\title{
The Temporal Confounding Effects of Extra-cerebral Contamination Factors on the Hemodynamic Signal Measured by Functional Near-Infrared Spectroscopy
}

\author{
Mehrdad Zarei ${ }^{1,2}$, Mohammad Ali Ansari ${ }^{2 *}$, Kourosh Zare ${ }^{1,2}$ \\ ${ }^{1}$ Laser Application in Medical Sciences Research Center, Shahid Beheshti University of Medical Sciences, Tehran, Iran \\ ${ }^{2}$ Laser and Plasma Research Institute, Shahid Beheshti University, Tehran, Iran
}

*Correspondence to Mohammad Ali Ansari, Assistant Professor of Biophotonics; Laser and Plasma Research Institute, Shahid Beheshti University, Velenjak, Tehran, Iran, 1983969411

Tel: +98-21-29904022; Email: m_ansari@sbu.ac.ir

Published online December 1, 2019

\begin{abstract}
Introduction: Functional near-infrared spectroscopy (fNIRS) has been broadly applied for optical brain imaging. This method is hemodynamic-based functional brain imaging relying on the measurement of the neurovascular coupling to detect changes in cerebral neuronal activities. The extra-cerebral hemodynamic changes are important contaminating factors in fNIRS measurements. This error signal can be misinterpreted as cerebral activities during fNIRS studies. Recently, it was assumed that temporal changes in deoxygenated hemoglobin concentration $[\mathrm{HHb}]$ was hardly affected by superficial blood flow, and it was proposed that the activation maps could be determined from $[\mathrm{HHb}]$ at large source-detector separation.

Methods: In the current study, we measured the temporal changes in [HHb] using a continueswave fNIRS device at large source-detector separation, while superficial blood flow was stimulated by infrared lasers. A mesh-based Monte Carlo code was applied to estimate fNIRS sensitivity to superficial hemodynamic changes in a realistic 3D MRI-based brain phantom.

Results: First, we simulated photon migration in a four-layered human-head slab model to calculate PPLs and fNIRS sensitivity. Then, the localization of the infrared laser inside a realistic brain model was studied using the Monte Carlo method. Finally, the changes in $[\mathrm{HHb}]$ over the prefrontal cortex of six adult males were measured by fNIRS at a source-detector separation of $3 \mathrm{~cm}$. The results demonstrated that the relation between fNIRS sensitivity and an increase in S-D separation was nonlinear and a correlation between shallow and deep signals was observed.

Conclusion: The presented results demonstrated that the temporal changes in the superficial blood flow could strongly affect $\mathrm{HHb}$ measurement at large source-detector separation. Hence, the cerebral activity map extracted from the $[\mathrm{HHb}]$ signal was mainly contaminated by superficial blood flow.

Keywords: Functional near-infrared spectroscopy; Photobiomodulation; Neurovascular coupling, Superficial blood flow.
\end{abstract}

\section{Introduction}

Functional near-infrared spectroscopy (fNIRS) as a noninvasive optical imaging method is increasingly applied to determine brain activity maps in cognitive studies, detect intracranial hemorrhage, and conduct transcranial monitoring in brain-injured adults. ${ }^{1-3}$ fNIRS devices measure cerebral blood flow (CBF) changes which may be linked to changes in cerebral neural activities or cerebral blood volume.

fNIRS, in contrast to fMRI, has high sensitivity to hemodynamic fluctuation in extra-cranial tissues. The changes in extra-cranial hemodynamic signals measured by fNIRS have been estimated to be over 10-20 times higher than the changes in cerebral tissue, depending on source-detector (S-D) separation. ${ }^{4}$ These superficial fluctuations attenuate cerebral hemodynamic responses (false negatives). In addition, the blood flow in the superficial tissues is affected by task-evoked changes in skin perfusion, body temperature, pain, cognitive and social stress, and emotions. ${ }^{5-8}$ These superficial taskrelated systemic changes can mimic the CBF changes that are called false positives. ${ }^{5}$ These false positives are major challenges decreasing the accuracy of continues- wave fNIRS (CW-fNIRS) measurements. Removing these false positives from CW-fNIRS is important because most of the clinical fNIRS studies have been implemented by CWfNIRS with a fixed $3 \mathrm{~cm}$ S-D separation. ${ }^{4,10}$

Several methods such as the statistical signal processing and the multi-distance (MD) method have been proposed to avoid or minimize false positives in CW-fNIRS measurements. In a part of these studies, researchers applied statistical and signal processing,

Please cite this article as follows: Zarei M, Ansari MA, Zare K. The temporal confounding effects of extra-cerebral contamination factors on the hemodynamic signal measured by functional near-infrared spectroscopy. J Lasers Med Sci. 2019;10(suppl 1):S73-S81. doi:10.15171/ jlms.2019.S14. 
for example, independent-component analysis (ICA), principal component analysis, general linear model, and adaptive filters to separate intracerebral and extracerebral signals from fNIRS signals. ${ }^{9,11-14}$ These methods often require additional measurements to extract the cerebral signal from fNIRS signals. Funane et al applied the multi-distance (MD) method, including shortdistance and long-distance detectors, and ICA to estimate extra-cranial hemodynamic. In addition, the authors emphasized that the scalp hemodynamics does not significantly change when the source-detector distance changes within the range of $10-40 \mathrm{~mm} .^{9}$ These studies rely on the assumption of a homogeneous spatial distribution of extra-cerebral changes in the superficial tissues. As presented in reference, ${ }^{14}$ this assumption is not accurate for the contribution of big arteries and veins supplying the scalp. Furthermore, some of these studies assumed that superficial signals had no correlation with cerebral signals. While Drummond et al, Kirilina et al, and Gilbert have shown the blood flow in the forehead skin is affected by the cognitive task, it is called systemic task-evoked hemodynamics. ${ }^{6,15,16}$

In addition to these types of statistical and signal processing, Tachtsidis and Scholkmann, in 2016, recommended that the effects of superficial scalp blood flow can be corrected by (i) calculating the mean $[\mathrm{HHb}]$ over all channels and using these channels as the superficial regressor and (ii) using short and long sourcedetector distance. ${ }^{5}$ Typically, the short-distance signal is scaled by a factor and subtracted from the long-distance signal. ${ }^{9,17}$ They stated that the effects of false positives on $[\mathrm{HHb}]$ measured at large S-D separation can be negligible compared with $\left[\mathrm{O}_{2} \mathrm{HH}\right]$. This assumption is based on the following points: (a) superficial hemodynamics does not significantly change when the source-detector separation changes within the range of $10-40 \mathrm{~mm},(b)$ there is a linear relation between an increase in S-D separation and fNIRS sensitivity, and $(c)$ the deoxygenated hemoglobin signal does not correlate with superficial artifacts at large S-D separation.

Kirilina et al used complementary time-domain fNIRS, fMRI, MR-angiography and peripheral physiological measurements (blood pressure, heart rate, skin conductance, and skin blood flow) to study activation in the frontal lobe during a continuous performance task. The authors applied fMRI scans to analyze fNIRS data, which included an additional predictor to determine systemic changes in the skin. ${ }^{6}$ They explained that the correlation coefficient between the predictors modeling extra-cranial and intracranial signals was less than 0.4 ; therefore, in this case, a fraction of the cerebral signal was removed. Moreover, they observed no obvious correlation between superficial artifacts and [HHb] changes.

This study aimed to estimate the contribution of superficial artifacts to the $[\mathrm{HHb}$ ] signals measured at large S-D separation. Hence, at first, we numerically evaluated the aforementioned assumptions applying for separating superficial signals from fNIRS signals, then the correlation between $[\mathrm{HHb}]$ and extra-cranial stimulation was estimated using fNIRS measurements. To do so, the 3D mesh-based Monte Carlo method developed by Fang, ${ }^{18}$ was used to simulate photon migration (lambda $760 \mathrm{~nm}$ ) in a four-layered human-head slab model to calculate fNIRS sensitivity because this parameter depends on the structural parameters, including tissue thickness and SCD, and the changes in superficial blood volume. We also studied the effect of the changes in SCD and the variation of blood volume in the cortical tissue on ANIRS sensitivity (in a 4-layer slab brain model). This planar model has been applied to calculate fNIRS sensitivity in the references ${ }^{19-21}$ because the estimation of fNIRS sensitivity in a realistic brain model can be complicated. In addition, the study of the effects of the changes in SCD thickness on fNIRS sensitivity in a realistic brain model is impossible. In the present study, we tried to focus on the extra-cranial signals and decrease the evoked and non-evoked cerebral signals using superficial blood flow (SBF) activation by photobiomodulation (SBFAP). To achieve this aim, we used photo-biostimulation to generate changes in the extra-cranial hemodynamics. The transcranial laser stimulation of the brain with near-infrared light is a novel form of photobiomodulation, or low-level laser therapy when applied to patients. ${ }^{22-26}$ In 2012, Rojas et al applied transcranial light stimulation (LED @ 660 nm) to adult male rats to facilitate cortical oxygenation and metabolic energy capacity. ${ }^{24}$ Tian et al first applied a continuous laser $\lambda=1064 \mathrm{~nm}$ to human transcranial laser stimulation, and a significant oxygenated hemodynamic was seen. ${ }^{25}$

In this study, in contrast to references. Tian et al and Hwang et al studies, ${ }^{25-26}$ we used a butterflied laser diode module $\lambda=1550 \mathrm{~nm}$ to stimulate superficial neuronal tissues because the absorption coefficients of brain tissues at $\lambda=1550 \mathrm{~nm}$ were larger than $\lambda=1064 \mathrm{~nm}$ and it significantly reduced the penetration depth of our laser inside the realistic brain. So, the majority of this laser might be absorbed by superficial neuronal tissues. Liljemalm et al, in 2013, applied a pulsed diode laser $(\lambda=$ $1550 \mathrm{~nm}$ ) launched into a 200-micron multimode optical fiber with a numerical aperture of 0.39 , and the radiant exposure was $5.2 \mathrm{~J} / \mathrm{cm}^{2}$ to stimulate rat cortical neurons in culture. ${ }^{27}$ Their measured neuronal activities were compatible with those presented in reference. ${ }^{28}$ In order to show that the majority of laser light was absorbed by superficial tissues, we simulated the propagation of this laser in a $3 \mathrm{D}$ realistic brain phantom using the meshbased Monte Carlo method to simulate the propagation of photons inside a well-known high-resolution Colin 27 template as an MRI-digital brain phantom. ${ }^{29}$ Then, we explored the effects of superficial contamination induced by SBFAP on the $[\mathrm{HHb}$ ] signal measured at large S-D separation. These results can be applied to estimate the contribution of superficial compartments to the CW- 
fNIRS signal, and these results can depict the significant relation between superficial hemodynamics and the fNIRS signal. The obtained results can also be applied to answer this question, "is it possible to purely separate extra-cranial and intracranial components from CWfNIRS signals at large S-D separation?" The answer can show an important factor limiting the accuracy of CWfNIRS measurements which have increasingly applied in clinical studies.

\section{Materials and Methods}

This study is divided into the numerical procedure and the experimental section. Here, we estimate the effects of superficial hemodynamics on the [HHb] signals. To do it, we first present concisely the Monte Carlo method in a turbid medium, and then the slab model, the 3D realistic brain model, and Colins 27 are introduced. Finally, the specifications of the infrared stimulator, the fNIRS device and the procedure of experiments are explained.

\section{Monte Carlo Method}

We employed a 3-D mesh-based Monte Carlo method to simulate laser propagation inside the brain as presented in Fang and Mahmoodkalayeh et al studies. ${ }^{18,30}$ Fifty million input photons were initially assigned and traced one by one as they propagated inside a brain model. The weight of each traveling photon decreased by a factor of $\exp \left(-\mu_{a} d\right)$, where $d$ is the distance traveled by the photon after each scattering. This distance is determined as $-\log (\zeta) /\left(\mu_{a}+\mu_{s}\right)$, where $\zeta$ is a random number less than a unit, and $\mu_{a}$ and $\mu_{s}$ are the absorption and scattering coefficients of the brain tissue respectively. The scattering angle $\theta$ is determined by the Henyey-Greenstein probability function ${ }^{31}$ :

$$
p_{H-G}(g, \theta)=\frac{1}{4 \pi} \frac{1-g^{2}}{\left(1+g^{2}-2 g \cos \theta\right)^{3 / 2}}
$$

\section{As follows:}

$$
\cos \theta=\frac{1}{2 g}\left\{1+g^{2}-\left[\frac{1-g^{2}}{1-g+g \xi}\right]\right\} \quad g \neq 0
$$

The parameter $g=\int p_{H-G}(g, \theta) \cos (\theta) d \Omega$, a number between -1 and 1 , describes the relative amount of the forward and backward scattering; for example, $g=0$ corresponds to an isotropic scattering and $g \rightarrow 1$ gives a highly forward scattering. This process continues until the photon exits from the brain model or is absorbed in a local region. This algorithm can yield the absorbed energy densities in each volume mesh inside the brain. Also, each Monte Carlo simulation stores the number of photons exiting through a $1.0 \mathrm{~mm}$ diameter of each detector location and the partial path length (PPL) traveled by that photon through each tissue layer. ${ }^{32,33}$

The sensitivity of fNIRS can be stated as the changes in the optical signal caused by a unit perturbation in the absorption coefficient of gray matter. It is usually expressed in optical density, $O D$ units $^{33,34}$ :

$\Delta O D=-\log \frac{I}{I_{0}}$

Where $I_{0}$ and $I$ are the baseline and the perturbed signal intensity respectively. The blood volume and oxygenation changes during functional brain activation cause changes in optical density. In literature and our Monte Carlo simulation method, only absorption changes are considered. The total optical signal measured at the scalp surface is a function of "mean distance" photons travelling from the source to the detector. This mean distance, or total path length (TPL), is also defined as the speed of light multiplied by mean total transit time for photons propagated in the medium. ${ }^{32}$ This mean distance is a set of PPLs, a distance a photon travels into each brain tissue layer such as the scalp, skull, cerebrospinal fluid (CSF), gray and white matter. The PPL (e.g. millimeters) derived by Hiraoka et al is as follows ${ }^{33}$ :

$\frac{\log \frac{I_{0}}{I}}{\delta \mu_{a, k}}=P P L_{k}$

$\delta \mu_{a, k}$ is a change in the absorption coefficient of layer $\mathrm{k}^{\text {th }}$. Based on the equation (4), the optical density change is given as:

$\Delta O D=\log \frac{I_{0}}{I}=\sum_{k=1}^{n} P P L_{k} \delta \mu_{a, k}$

So, $P P L_{k}$ represents the sensitivity to absorption changes in tissue $k$. The TPL is also estimated by:

$$
T P L=\sum_{k=1}^{n} P P L_{k}
$$

Hence, the relative sensitivity is given as $P P L_{k} / T P L$

Brain Model

We simulated the contributions of the superficial blood changes in fNIRS sensitivity to the cortical signals using the Monte Carlo method. Moreover, the effects of CSF thickness on fNIRS sensitivity were studied on a left frontal pole, FP1, according to the international 10-20 system (see Figure 1A). Our fNIRS system, as explained later, applied near-infrared light sources to measure hemodynamic signals $\left([\mathrm{HHb}]\right.$ and $\left.\left[\mathrm{O}_{2} \mathrm{HH}\right]\right)$. Therefore, we simulated near-infrared photon migration in a fourlayered human-head slab model (Figure 2), which included scalp and skull, CSF, and gray and white matter. The optical properties and tissue thicknesses were given to be similar to FP1 as depicted in Table 1 (these values were represented as the mean value published in Fang and Ansari et al studies ${ }^{18,31}$ ).

Then, we simulated the absorption of infrared laser energy inside a realistic brain phantom by the mesh- 

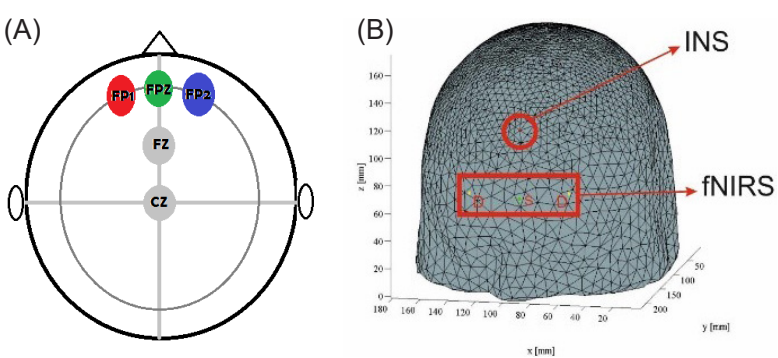

Figure 1. The Locations of FP1, FP2, and FPZ in the International 10-20 System (a), and the Position of IR Laser Stimulation (INS) and fNIRS Probs (b).

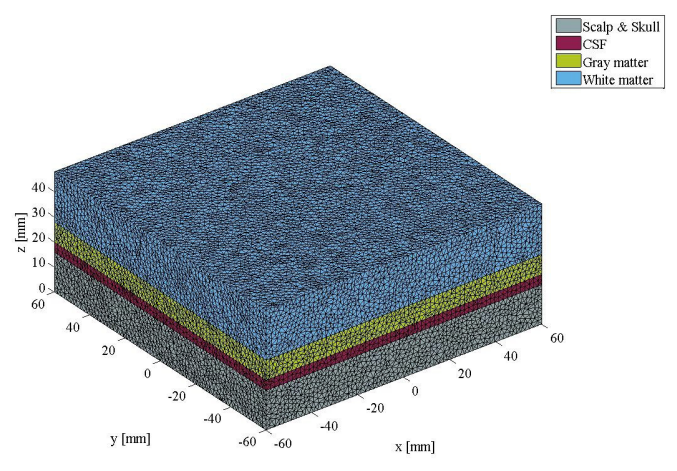

Figure 2. The Simulation Model of a Human Head Having 4 Layers: Skull, CSF, Gray Matter, and White Matter.

Table 1. Optical Properties and Thicknesses of Tissue Layers in the 4-Layered Human-Head Slab Model (as depicted in Figure 2) at a Wavelength of 760 $\mathrm{nm}$

\begin{tabular}{lcccc}
\hline Tissue Type & $\boldsymbol{\mu}_{\mathbf{a}}\left(\mathbf{c m}^{-1}\right)$ & $\boldsymbol{\mu}_{\mathbf{s}}\left(\mathbf{c m}^{-1}\right)$ & $\mathbf{g}$ & Thickness $(\mathbf{c m})$ \\
\hline Scalp and skull & 0.19 & 78.2 & 0.89 & 1.57 \\
CSF & 0.02 & 0.1 & 0.89 & 0.30 \\
Gray matter & 0.20 & 90 & 0.89 & 0.80 \\
White matter & 0.80 & 409.0 & 0.89 & 2.00 \\
\hline
\end{tabular}

based Monte Carlo method. To do so, we used a wellknown $1 \times 1 \times \mathrm{mm}^{3}$ Colin 27 brain template (see Figure 3). This template included scalp and skull, CSF, and gray and white matter. ${ }^{34,35}$ The optical properties of these tissue types at a wavelength of $1550 \mathrm{~nm}$ were assigned as per Table 2.

\section{Experimental Set-up}

In this study, we used an infrared pulsed butterfly laser module at a wavelength of $1550 \mathrm{~nm}$ for SBFAP in the middle of the FZ-FPZ line (see Figure 1A). The laser pulse was delivered to the cap via a 400-micron multimode optical fiber $(\mathrm{NA}=0.39)$ as depicted in Figure 1B. The radiant exposure of the infrared laser was adjusted to about $2.3 \mathrm{~J} / \mathrm{cm}^{2}$ to avoid physical damage and tissue
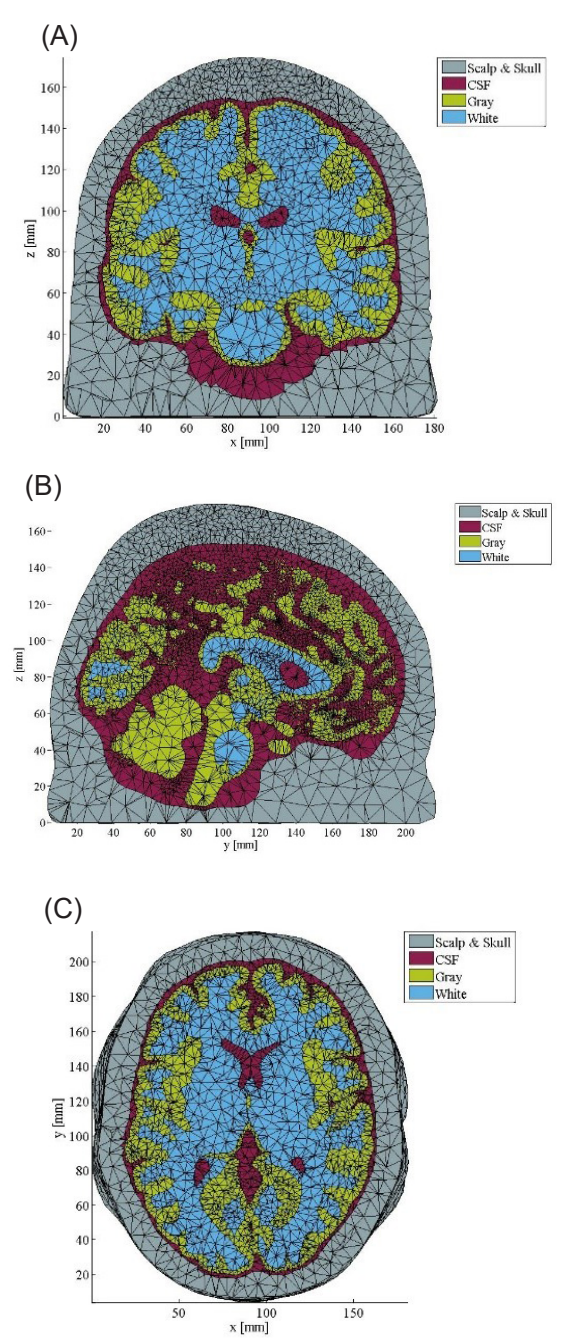

Figure 3. Segmented Colin 27 Head Model (Coronal, Sagittal, and Transverse Slides).

Table 2. Optical Properties for the scalp, CSF, Gray Matter, and White Matter During Monte Carlo Simulation at a Wavelength of $1550 \mathrm{~nm}$

\begin{tabular}{lccc}
\hline Tissue Type & $\boldsymbol{\mu}_{\mathrm{a}}\left(\mathbf{c m}^{-1}\right)$ & $\boldsymbol{\mu}_{\mathbf{s}}\left(\mathbf{c m}^{-1}\right)$ & $\mathbf{g}$ \\
\hline Scalp and skull & 15.00 & 65.23 & 0.84 \\
CSF & 15.00 & 0.09 & 0.84 \\
Gray matter & 15.00 & 69.43 & 0.84 \\
White matter & 15.00 & 37.27 & 0.84 \\
\hline
\end{tabular}

The refractive index is assumed to be 1.37. Due to high laser absorption by the water content in these tissue layers, the absorption coefficient $\mu_{\mathrm{a}}$ is similar to the absorption coefficient of water

heating. The repetition rate and the pulse duration were 6 $\mathrm{Hz}$ and $20 \mathrm{~ms}$ respectively.

Each experiment included a 180-second baseline as a rest period, 60-second infrared neural stimulation, and finally an 80 -second post-test rest period. The changes in $[\mathrm{HHb}]$ and $\left[\mathrm{O}_{2} \mathrm{HH}\right]$ over the left frontal pole (FP1) and right frontal pole (FP2) during the superficial infrared neural stimulation were measured by the portable 
wireless four-channel fNIRS. This system uses a NIR diode EPITEX (L4*730/4*805/4*850) as light sources and four OPT101 as detectors. The data sampling rate was $144 \mathrm{~Hz}$. The source-detector separation was $3 \mathrm{~cm}$.

Six healthy adult male participants whose ages ranged from 21 to 25 were recruited from the Shahid Beheshti University. Also, they did not have and were not suffering from any psychiatric disorder. Informed consent was obtained prior to each experiment. All of the laser safety guidelines were followed during the experiments. Experiments were conducted in a locked room with no reflective surfaces. During the experiments, the participants were instructed to keep their eyes closed during laser stimulation.

All fNIRS data after the removal motion artifacts were processed by ANOVA method to extract the $[\mathrm{HHb}]$ and $\left[\mathrm{O}_{2} \mathrm{HH}\right]$.

\section{Results}

The aim of this study was the estimation of the effects of the superficial hemodynamic signal (false positives) on the $[\mathrm{HHb}]$ signal measured at large S-D separation.

First, we simulated photon migration (lambda $760 \mathrm{~nm}$ ) in a four-layered human-head slab model to calculate PPLs and fNIRS sensitivity. Then, the localization of the infrared laser inside Colins 27 was studied using Monte Carlo method. Finally, the effects of superficial hemodynamics induced by SBFAP on the [HHb] signals were estimated.

Figure $4 \mathrm{~A}$ illustrates the contribution of each layer to the CW-fNIRS signal as a function of S-D separation. This figure illustrates that (i) more than $80 \%$ of the measured fNIRS signal corresponds to extra-cranial components for $S$-D larger than $3 \mathrm{~cm}$, namely the superficial signals which are approximately 20 times larger than cortical signals and (ii) the cortical signals nonlinearly increase along with an increase in S-D distance. In addition, an increase in SCD (accompanied by an increase in the thickness of CSF) can change fNIRS sensitivity, as illustrated in Figure 4B. In order to study the contribution of the changes in the superficial blood volume to sensitivity, we assumed that these changes corresponded to the changes in the absorption coefficient of scalp and skull. Figure $4 \mathrm{C}$ depicts that $\triangle O D$ measured by fNIRS significantly increases by superficial blood volume changes. This increase in $\triangle O D$, at large S-D separation, can be misinterpreted as cortical activities. These results demonstrate that the relation between fNIRS sensitivity and an increase in S-D separation is nonlinear and one can also see a correlation between shallow and deep signals. These results also show that using short-distance scaling can be a contaminated factor decreasing the accuracy of fNIRS measurements.

To show a relation between [HHb] signals and superficial hemodynamics, a low-level infrared laser $($ lambda $=1550 \mathrm{~nm})$ was applied to shine the brain frontal cortex for stimulating extracerebral neuronal tissues. Then, the absorption of laser light in a 3D realistic human brain was simulated by the mesh-based Monte Carlo method. We traced the propagation of 50 million photons inside the 3D realistic brain phantom, Colin 27 adult brain template and it took more than 190 minutes to run on a Xeon $2.4 \mathrm{GHz}, 16 \mathrm{~GB}$ RAM. The laser energy absorbed by brain tissues is depicted in Figure 5 (coronal, sagittal, and transverse slides). These results illustrate that the majority of the laser light is localized in the scalp and skull close to the illumination point. The energy deposited in extracerebral tissues caused a temperature increase of about $10 \mathrm{mK}$. The small penetration depth and the low-temperature increase confirm that cortical neurons cannot be excited by this infrared laser. During SBFAP, a portable fNIRS was placed on participant's foreheads to

(A)

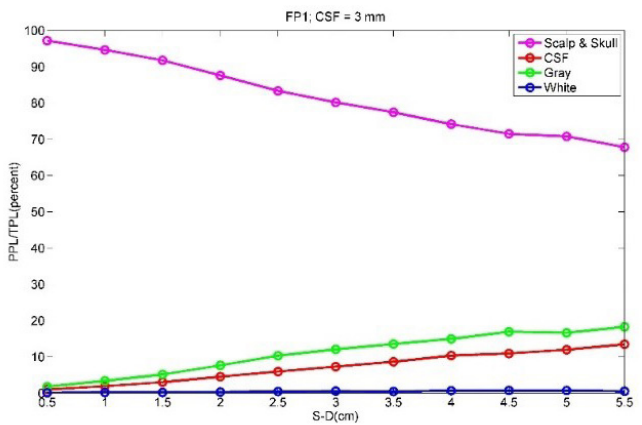

(B)

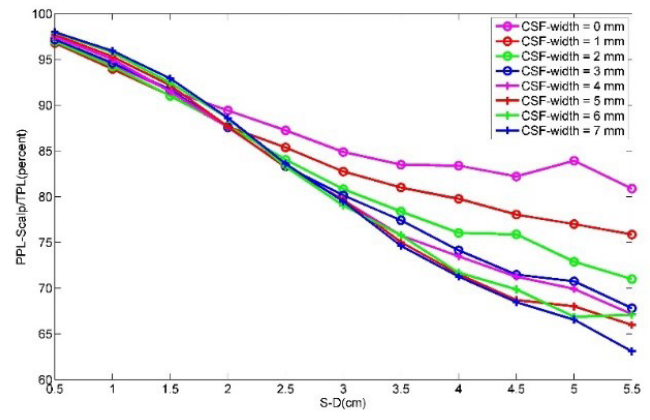

(C)

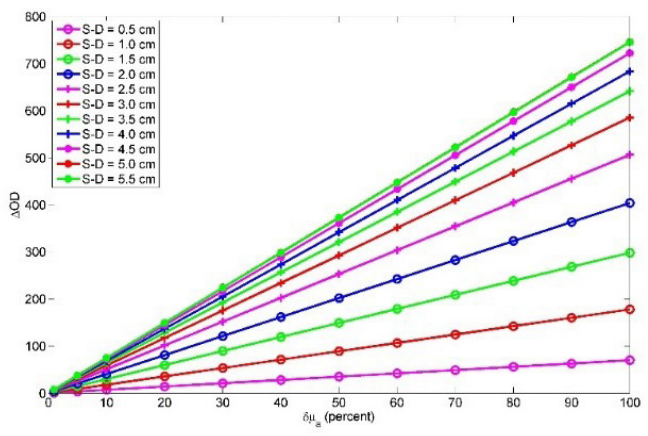

Figure 4. The Contribution of Brain Layers to the fNIRS Signal. (A) the relative sensitivity of brain tissues, (B) the variation of scalp PPLs for different CSF thicknesses, and (C) changes in the fNIRS signal at the location of FP1, given $1 \%-100 \%$ increase in the scalp absorption coefficient, plotted by S-D separation. 
(A)

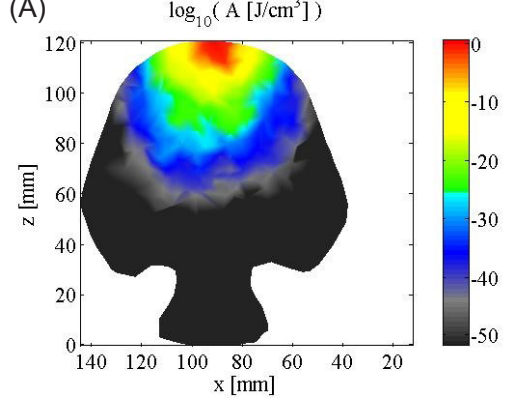

(B)

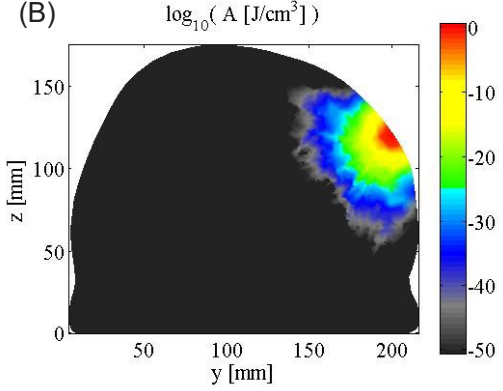

(C)

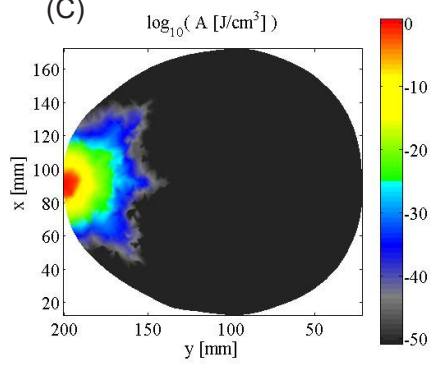

Figure 5. The Distribution of the Laser Deposited Inside the Colin 27 Template (lambda=1550 nm) in Coronal (Aa), Sagittal (B), Transverse (C) Slides.

measure changes in hemodynamic signals as depicted in Figure 6 . The changes in $[\mathrm{HHb}]$ and $\left[\mathrm{O}_{2} \mathrm{HH}\right]$, the average rise time from the baseline to the maximum, and recovery time to return to the baseline are depicted in Table 3.

\section{Discussion and Conclusion}

Recent fNIRS- fMRI studies have demonstrated that frontal/forehead fNIRS measurements are strongly contaminated with superficial forehead veins., ${ }^{5,37}$ Moreover, it has been revealed that the SDC increases in the medial frontal region compared to the more lateral frontal area. ${ }^{16}$ Liebert et al, Kirilina et al, and Haeussinger et al state that the prefrontal fNIRS measurements are about twenty times more sensitive to SBF than $\mathrm{CBF}^{4,6,10}$ It is often assumed that for short S-D distance (i.e. $<1.0$ $\mathrm{cm}$ ) the fNIRS signals exclusively consist of extra-cranial compartments which are used for SBF correction. However, due to inhomogeneity of forehead blood regulation, using this correction to remove SBF from fNIRS significantly reduces the accuracy of fNIRS measurements. Therefore, estimating the contribution of SBF to the fNIRS signal - or measuring the superficial PPL - is an essential step to improve fNIRS measurements. Measuring the superficial PPL can be conducted by time-resolved fNIRS in which the signal of early photons mainly reflects SBF. Aletti et al have shown that time-resolved fNIRS can be used to distinguish between intracranial and extracranial signals. ${ }^{36}$ As mentioned in the introduction section, Kirilina et al applied fMRI to determine the origins of superficial signals. However, superficial arteries might not be visible in the fMRI due to the low partial volume of blood and short relaxation time in these compartments. ${ }^{6}$

According to reference, ${ }^{5}$ we have shown that for large S-D distance, the superficial PPLs are about 20 times larger than the cerebral PPL (see Figure 4a). In other words, the fronto-polar fNIRS signal generally consists of the SBF changes.

In this study, regardless of the short-distance approach, we proposed an easy method to measure changes in the superficial hemodynamic signals. The superficial tissues of the medial frontal region were stimulated by SBFAP. The mesh-based Monte Carlo method was used to

(A)

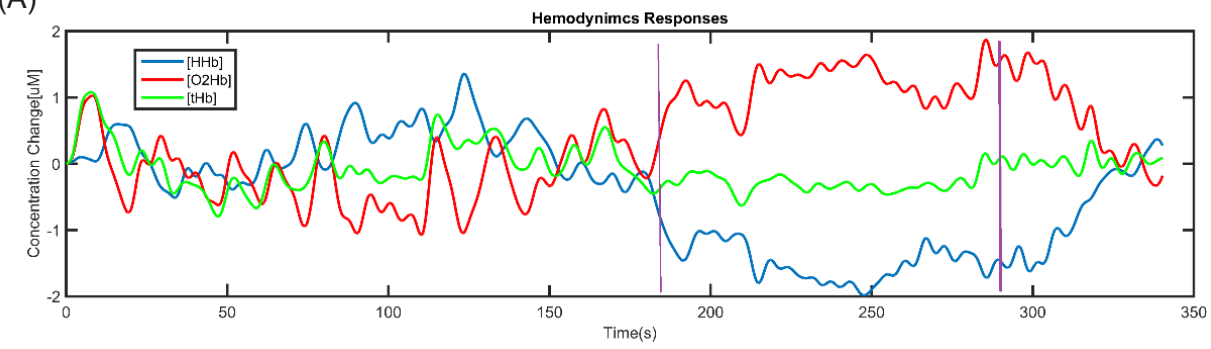

(B)

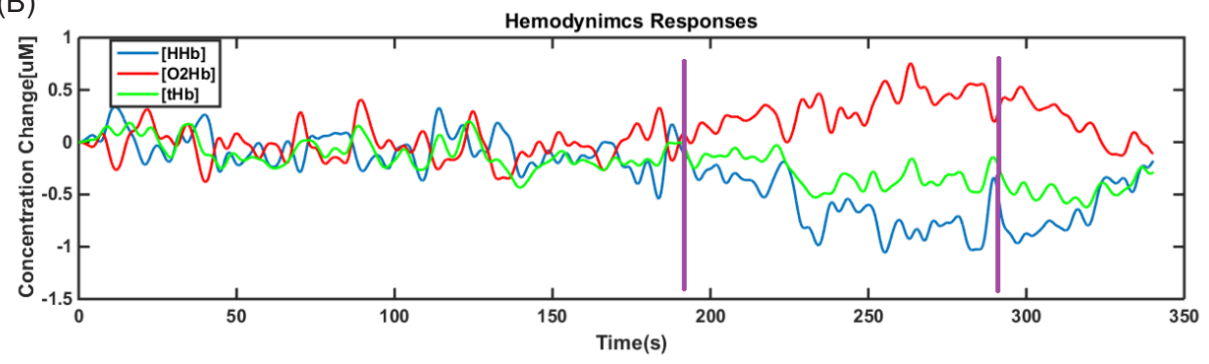

Figure 6. Prefrontal Hemodynamic Responses Induced by Infrared Neural Stimulation in (a) the Left and (b) Right Prefrontal Cortex. 
Table 3. Estimated Changes in Deoxygenated Hemoglobin [HHb], Oxygenated Hemoglobin $\left[\mathrm{O}_{2} \mathrm{HH}\right], \mu \mathrm{M}$, and Their Rise Time and Recovery Time for Left and Right Channels

\begin{tabular}{|c|c|c|c|c|c|c|c|c|c|c|c|c|}
\hline $\begin{array}{l}\text { No. of } \\
\text { Subject }\end{array}$ & $\begin{array}{c}\text { Left } \\
{[\mathrm{HHb}]}\end{array}$ & $\begin{array}{l}\text { Rise Time } \\
\text { (s) }[\mathrm{HHb}]\end{array}$ & $\begin{array}{c}\text { Recovery } \\
\text { Time (s) } \\
{[\mathrm{HHb}]}\end{array}$ & $\begin{array}{c}\text { Left } \\
{\left[\mathrm{O}_{2} \mathrm{HH}\right]}\end{array}$ & $\begin{array}{l}\text { Rise Time } \\
\text { (s) }\left[\mathrm{O}_{2} \mathrm{HH}\right]\end{array}$ & $\begin{array}{c}\text { Recovery } \\
\text { time (s) } \\
{\left[\mathrm{O}_{2} \mathrm{HH}\right]}\end{array}$ & $\begin{array}{l}\text { Right } \\
{[\mathrm{HHb}]}\end{array}$ & $\begin{array}{c}\text { Rise } \\
\text { Time (s) } \\
{[\mathrm{HHb}]}\end{array}$ & $\begin{array}{c}\text { Recovery } \\
\text { Time (s) } \\
{[\mathrm{HHb}]}\end{array}$ & $\begin{array}{c}\text { Right } \\
{\left[\mathrm{O}_{2} \mathrm{HH}\right]}\end{array}$ & $\begin{array}{c}\text { Rise } \\
\text { Time (s) } \\
{\left[\mathrm{O}_{2} \mathrm{HH}\right]}\end{array}$ & $\begin{array}{c}\text { Recovery } \\
\text { Time (s) } \\
{\left[\mathrm{O}_{2} \mathrm{HH}\right]}\end{array}$ \\
\hline 1 & -0.5 & 68 & 68 & 0.6 & 50 & 65 & -0.4 & 50 & 68 & 0.3 & 50 & 65 \\
\hline 2 & -0.6 & 70 & 65 & 0.6 & 55 & 70 & -0.5 & 52 & 70 & 0.6 & 60 & 70 \\
\hline 3 & -1.0 & 80 & 65 & 1.1 & 50 & 65 & -0.9 & 70 & 80 & 0.6 & 70 & 80 \\
\hline 4 & -0.1 & 65 & 70 & 1.2 & 55 & 68 & -0.5 & 65 & 65 & 0.8 & 55 & 75 \\
\hline 5 & -0.5 & 75 & 65 & 0.6 & 50 & 65 & -0.6 & 65 & 75 & 0.4 & 70 & 70 \\
\hline 6 & -0.1 & 75 & 65 & 0.8 & 60 & 70 & -0.2 & 68 & 75 & 0.6 & 75 & 70 \\
\hline
\end{tabular}

simulate the distribution of absorbed laser energy inside the Colins 27 template. Figure 5 illustrates that the IR laser was localized inside the superficial tissue. We also applied an electroencephalography (EEG) experiment to convince that cerebral tissues were not excited by this lowlevel INS, and the prefrontal fNIRS signals were measured at the $\mathrm{S}-\mathrm{D}$ separation of $3 \mathrm{~cm}$. Using CW-fNIRS, we could solely measure the frontopolar superficial hemodynamic changes. This amount could also be used to estimate the superficial PPL.

The presented results in Table 3 demonstrate that the SBFAP in the middle of the FPZ-FZ line caused changes in the hemodynamic signal measured in FP1 and FP2. As depicted in Figure1, the distance between the location of SBFAP and detectors was larger than $3 \mathrm{~cm}$. We also asked the participants to relax during the test. The illustrated results in Figure5 demonstrate that deposited energy was localized and the energy density decreased by 20 orders at a distance of $2 \mathrm{~cm}$ from the FPZ-FZ line, while a significant change in $[\mathrm{HHb}]$ during SBFAP was seen. The depicted results in Table 3 illustrate that the temporal changes in $[\mathrm{HHb}]$ and $\left[\mathrm{O}_{2} \mathrm{HH}\right]$ induced by SBFAP correlated with the absolute Pearson's correlation coefficient of 0.86 . The changes in hemodynamic signals were greater than those obtained in references ${ }^{5,9}$ because using short-distance scaling led to an underestimation of the true hemodynamic signals. ${ }^{5}$ The rise time of [HHb] during SBFAP correlated with the changes in $[\mathrm{HHb}]$ with a Pearson correlation coefficient of 0.70 . The long rise time and recovery time of the changes in $[\mathrm{HHb}]$ show that SBFAP has an important impact on the fNIRS signals as a false positive artifact. Hence, for large S-D separation, $\mathrm{SBF}$ can influence the $[\mathrm{HHb}]$ signals.

Heinzel et al and Kirilina et al have demonstrated that the temporal changes in $[\mathrm{HHb}]$ are hardly affected by SBF. Haeussinger et al. have also applied this assumption to improve the fNIRS signals. ${ }^{10,38}$ Kirilina et al, in 2012, observed a pronounced artifact for oxygenated hemoglobin but not for deoxygenated hemoglobin, and this may provide a tool to avoid false positives in the fNIRS activation map, or more precisely, to only determine the activation maps from the changes in $[\mathrm{HHb}] .{ }^{6}$ It was often assumed that the signal from shallow layers (shallow signal) had no correlation with the signal from deep tissues (deep signal), and the fNIRS signal was a linear combination of the shallow and deep signals.

Hiraoka et al in 1993 and Kohri et al in 2002 assumed that the PPL of the superficial tissue layer was constant for an S-D distance of greater than $10 \mathrm{~mm} \cdot{ }^{33,39}$ This assumption was evaluated by a Monte Carlo simulation by Yamada et al in 2009. In the study, they applied a four-layered optical slab model of an adult head, which consisted of the layers of scalp and skull, CSF, gray and white matter. ${ }^{40}$ Another assumption of theirs was that the temporal change in the scattering and absorption was uniform in each layer. While these assumptions led to a decrease in the accuracy of prefrontal CW-fNIRS measurements, our results showed that SBF had a significant effect on [HHb] measured at a large S-D separation, and it was consequently impossible to purely separate extra-cerebral and cerebral components from CW-fNIRS.

The results also present a limitation for brain computer interfaces and medical applications based on prefrontal CW-fNIRS. It seems prefrontal CW-fNIRS (with a large S-D separation) cannot be applied solely to determine the prefrontal brain activity maps.

\section{Ethical Considerations}

Not applicable.

\section{Conflict of Interests}

The authors declare that they have no competing interests.

\section{Acknowledgment}

We thank Dr. Reza Khosrowabadi for his helpful contribution to our discussions and his support in the EEG measurements.

\section{References}

1. Ansari MA, Zakeri M. Blind localization of heating in neural tissues induced by a train of infrared pulse laser. J Lasers Med Sci. 2019;10(4):264-67. 
doi:10.15171/jlms.2019.43.

2. Salonia R, Bell M), Kochanek PM, Berger RP. The utility of near-infrared spectroscopy indetecting intracranial hemorrhage in children. J Neurotrauma. 2012;29(6):1047-53. doi: 10.1089/neu.2011.1890.

3. Kim MN, Durduran T, Frangos S, Edlow BL, Buckley EM, Moss HE, et al. Noninvasive measurement of cerebral blood flow and blood oxygenation using near-infrared and diffuse correlation spectroscopies in critically brain-injured adults. Neurocrit Care. 2010;12(2):173-80. doi:10.1007/s12028-009-9305-x.

4. Liebert A, Wabnitz H, Steinbrink J, Obrig H, Möller M, Macdonald R, et al. Time-resolved multidistance near-infrared spectroscopy of the adult head: intracerebral and extracerebral absorption changes from moments of distribution of times of flight of photons. Appl Opt.2004;43(15):3037-47. doi:10.1364/ ao.43.003037.

5. Tachtsidis I, Scholkmann H. False positives and false negatives in functional near-infrared spectroscopy: issues, challenges, and the way forward. Neurophotonics. 2016;3(3):031405. doi:10.1117/1. NPh.3.3.039801.

6. Kirilina E, Jelzow A, Heine A, Niessing M, Wabnitz $\mathrm{H}$, Brühl R, et al. The physiological origin of taskevoked systemic artefacts in functional near infrared spectroscopy. Neuroimage.2012;61(1):70-81. doi:10.1016/j.neuroimage.2012.02.074.

7. Yamada T, Umeyama S, Matsuda K. Separation of fNIRS signals into functional and systemic components based on differences in hemodynamic modalities. PloS One. 2012;7(11):e50271. doi:10.1371/ journal.pone.0050271.

8. Scholkmann F, Kleiser S, Metz AJ, Zimmermann R, Mata Pavia J, Wolf $\mathrm{U}$, et al. A review on continuous wave functional near-infrared spectroscopy and imaging instrumentation and methodology. Neuroimage. 2014;85(1): 6-27. doi:10.1016/j. neuroimage.2013.05.004.

9. Funane T, Atsumori H, Katura T, Obata AN, Sato H, Tanikawa Y, et al. Quantitative evaluation of deep and shallow tissue layers' contribution to fNIRS signal using multi-distance optodes and independent component analysis. Neuroimage. 2014;85(1):150-65. doi:10.1016/j.neuroimage.2013.02.026.

10. Haeussinger FB, Dresler T, Heinzel S, Schecklmann $\mathrm{M}$, Fallgatter AJ, Ehlis AC. Reconstructing functional near-infrared spectroscopy (fNIRS) signals impaired by extra-cranial confounds: an easy-to-use filter method. Neuroimage. 2014;95:69-79. doi: 10.1016/j. neuroimage.2014.02.035.

11. Cooper RJ, Selb J, Gagnon L, Phillip D, Schytz HW, Iversen $\mathrm{HK}$, et al. A systematic comparison of motion artifact correction techniques for functional nearinfrared spectroscopy. Front Neurosci. 2012;6:147. doi:10.3389/fnins.2012.00147.
12. Medvedev AV, Kainerstorfer J, Borisov SV, Barbour RL, VanMeter J. Event-related fast optical signal in a rapid object recognition task: improving detection by the independent component analysis. Brain Res. 2008;1236:145-58. doi: 10.1016/j. brainres.2008.07.122.

13. Gagnon L, Yücel MA, Boas DA, Cooper RJ. Further improvement in reducing superficial contamination in NIRS using double short separation measurements. Neuroimage. 2014;85(1):127-35. doi:10.1016/j. neuroimage.2013.01.073.

14. Gagnon L, Cooper RJ, Yücel MA, Perdue KL, Greve DN, Boas DA. Short separation channel location impacts the performance of short channel regression in NIRS. Neuroimage. 2012;59(3):2518-28. doi:10.1016/j.neuroimage.2011.08.095.

15. Drummond PD, Lazaroo D. The effect of facial blood flow on ratings of blushing and negative affect during an embarrassing task: Preliminary findings. J Anxiety Disord. 2012;26(2):305-10. doi:10.1016/j. janxdis.2011.12.012.

16. Gilbert SJ, Spengler S, Simons JS, Steele JD, Lawrie SM, Frith CD , et al. Functional specialization within rostral prefrontal cortex (area 10): a meta-analysis. J Cogn Neurosci. 2006;18(6):932-48. doi:10.1162/ jocn.2006.18.6.932.

17. Koenraadt KL, Roelofsen EG, Duysens J, Keijsers NL. Cortical control of normal gait and precision stepping: an fNIRS study. Neuroimage. 2014;85(1):415-22. doi: 10.1016/j.neuroimage.2013.04.070.

18. Fang Q. Mesh-based Monte Carlo method using fast ray-tracing in Plücker coordinates. Biomed Opt Express. 2010;1(1):165-75. doi:10.1364/ BOE.1.000165.

19. Wang S, Shibahara N, Kuramashi D, Okawa S, Kakuta N, Okada E, et al. Effects of spatial variation of skull and cerebrospinal fluid layers on optical mapping of brain activities. Opt Rev. 2010;17(4):410-420. doi:10.1007/s10043-010-0076-6.

20. Okada E, Delpy DT. Near-infrared light propagation in an adult head model. I. Modeling of low-level scattering in the cerebrospinal fluid layer. Appl Opt. 2003;42(16):2906-14. doi:10.1364/ao.42.002906.

21. Farina A, Torricelli A, Bargigia I, Spinelli L, Cubeddu $\mathrm{R}$, Foschum $\mathrm{F}$, et al. In-vivo multilaboratory investigation of the optical properties of the human head. Biomed Opt Express. 2015;6(7):2609-23. doi:10.1364/BOE.6.002609.

22. Tedford CE, DeLapp S, Jacques S, Anders J. Quantitative analysis of transcranial and intraparenchymal light penetration in human cadaver brain tissue. Lasers Surg Med. 2015;47(4):312-22. doi:10.1002/lsm.22343.

23. Konstantinović LM, Jelić MB, Jeremić A, Stevanović VB, Milanović SD, Filipović SR. Transcranial application of near-infrared low-level laser can 
modulate cortical excitability. Lasers Surg Med. 2013;45(10):648-53. doi:10.1002/lsm.22190.

24. Rojas JC, Bruchey AK, Gonzalez-Lima F. Lowlevel light therapy improves cortical metabolic capacity and memory retention. J Alzheimer Dis. 2012;32(3):741-52. doi: 10.3233/JAD-2012-120817.

25. Tian F, Hase SN, Gonzalez-Lima F, Liu H. Transcranial laser stimulation improves human cerebral oxygenation. Lasers Surg Med. 2016;48(4):343-9. doi:10.1002/lsm.22471.

26. Hwang J, Castelli DM, Gonzalez-Lima F. Cognitive enhancement by transcranial laser stimulation and acute aerobic exercise. Lasers Med Sci. 2016;31(6):1151-60. doi: 10.1007/s10103-016-19623.

27. Liljemalm R, Nyberg $T$, von Holst $H$. Heating during infrared neural stimulation. Lasers Surg Med. 2013;45(7):469-81. doi:10.1002/lsm.22158.

28. Wells J, Konrad P, Kao C, Jansen ED, MahadevanJansen A. Pulsed laser versus electrical energy for peripheral nerve stimulation. J Neurosci Methods. 2007;163(2):326-37. doi:10.1016/j. jneumeth.2007.03.016.

29. Collins DL, Zijdenbos AP, Kollokian V, Sled JG, Kabani NJ, Holmes CJ, et al. Design and construction of a realistic digital brain phantom. IEEE Trans Med Imaging. 1998;17(3):463-8. doi:10.1109/42.712135.

30. Mahmoodkalayeh S, Ansari MA, Tuchin VV. Head model based on the shape of the subject's head for optical brain imaging. Biomed Opt express. 2019;10(6):2795-2808. doi: 10.1364/BOE.10.002795.

31. Ansari MA, Massudi R, Hejazi M. Experimental and numerical study on simultaneous effects of scattering and absorption on fluorescence spectroscopy of a breast phantom. Opt Laser Technol. 2009;41(6):74650. doi:10.1016/j.optlastec.2008.12.019.

32. Strangman GE, Zhang Q, Li Z. Scalp and skull influence on near infrared photon propagation in the Colin27 brain template. Neuroimage. 2014;85(1):13649. doi: 10.1016/j.neuroimage.2013.04.090.

33. Hiraoka M, Firbank M, Essenpreis M, Cope M, Arridge
SR, Van der Zee P,et al. A Monte Carlo investigation of optical pathlength in inhomogeneous tissue and its application to near-infrared spectroscopy. Phys Med Biol. 1993;38(12):1859-76. doi:10.1088/00319155/38/12/011.

34. Ansari MA, Erfanzadeh M, Mohajerani E. Mechanisms of laser-tissue interaction: II. Tissue thermal properties. J Lasers Med Sci. 2013;4(3): 99106. doi:10.22037/jlms.v4i3.4681.

35. Ansari MA, Zarei M, Akhlagipour N, Niknam AR. Skull and cerebrospinal fluid effects on microwave radiation propagation in human brain. J Phys D Appl Phys. 2017;50(49):495401. doi:10.1088/1361-6463/ aa944b.

36. Cui X, Bray S, Bryan DM, Glover GH, Reiss AL. A quantitative comparison of NIRS and fMRI across multiple cognitive tasks. Neuroimage. 2011;54(4):2808-21. doi: 10.1016/j. neuroimage.2010.10.069.

37. Aletti F, Re R, Pace V, Contini D, Molteni E, Cerutti $S$, et al. Deep and surface hemodynamic signal from functional time resolved transcranial near infrared spectroscopy compared to skin flowmotion. Comput Biol Med. 2012;42(3):282-9. doi: 10.1016/j. compbiomed.2011.06.001.

38. Heinzel S, Haeussinger FB, Hahn T, Ehlis AC, Plichta MM, Fallgatter AJ. Variability of (functional) hemodynamics as measured with simultaneous fNIRS and fMRI during intertemporal choice. Neuroimage. 2013;71:125-34. doi:10.1016/j. neuroimage.2012.12.074.

39. Kohri S, Hoshi Y, Tamura M, Kato C, Kuge Y, Tamaki N. Quantitative evaluation of the relative contribution ratio of cerebral tissue to near-infrared signals in the adult human head: a preliminary study. Physiol Meas. 2002;23(2):301-12. doi:10.1088/0967-3334/23/2/306.

40. Yamada T, Umeyama S, Matsuda K. Multidistance probe arrangement to eliminate artifacts in functional near-infrared spectroscopy. J Biomed Opt. 2009;14(6):064034. doi: 10.1117/1.3275469. 